\title{
Substance Use Dose Form
}

National Cancer Institute

\section{Source}

National Cancer Institute. Substance Use Dose Form. NCI Thesaurus. Code C83417.

The dosage form of a substance in use. 